\title{
Modeling of a Mobile Manipulator for Redundancy Resolution
}

\author{
Baeksuk Chu $^{1 *}$ \\ ${ }^{1}$ Department of Intelligent Mechanical Engineering, Kumoh National Institute of Technolo- \\ gy, Gyeongbuk, Korea \\ *Corresponding author (bschu@kumoh.ac.kr)
}

\begin{abstract}
Purpose Recently, various applications of robotic systems to construction sites have been tried. In this research, a mobile platform-based serial manipulator, the so called 'mobile manipulator', is employed for construction tasks such as material handling, beam assembly, welding, and so on. Generally, a serial manipulator has 6 DOF (degrees of freedom). The mobile manipulator suggested in this paper adds 2 or $3 \mathrm{DOF}$, so that there are a total of 8 or 9 DOF. In 3-dimensional space, the minimum DOF for manipulation is 6 . A manipulator that has more DOF than 6 is called a manipulator with 'redundancy'. While a manipulator with redundancy like the proposed mobile manipulator has several advantages such as large flexibility, singularity avoidance, easy obstacle avoidance, and so on, the redundancy resolution for optimal manipulation is not easy. In this research, modeling of the mobile manipulator for redundancy resolution is studied. Method Since the suggested mobile manipulator is a MIMO(multi-input multi-output) robot system with 8 or 9 links, a state-space model is derived for redundancy resolution. Analysis of kinematics and dynamics for a serial manipulator and a mobile platform is performed. An integration of the serial manipulator and the mobile platform is used. Results \& Discussion In this research, a state-space model for the proposed mobile manipulator is derived. This model enables redundancy resolution for optimal solution of 8 or 9 DOF-robot system. In order to solve the redundancy resolution problem, a linear quadratic method, which is one of the conventional optimal control methods, or a reinforcement learning method can be used.
\end{abstract}

Keywords: mobile manipulator, redundancy resolution, construction robot

\section{INTRODUCTION AND PRELIMINARIES}

Modern robot systems have broadened their working capacity and application areas by fully or partially automating industrial systems, especially manufacturing, assembly, palletizing, and so on, or cooperating such tasks with laborers. So far, robotic systems have been dominated by serial-typed manipulators with a fixed platform. However, as working process and configuration had been complicated and the types of tasks had significantly increased, conventional manipulatory process which had been performed on a fixed base encountered a marginal situation in aspect of cost and productivity. Recently, a novel manipulatory alternative has appeared, whose name is mobile manipulator which combines a robotic manipulator with a mobile platform. It contains a mobile capacity so that it moves several working sites and extends the working space of the manipulator infinitely. Palletizing process of huge elements such as Flat Panel Display (FPD) is one of the representative applications of the mobile manipulator, where a serial manipulator for panel handling is combined with a longitudinal mobile cart for transferring the manipulator and the panel. Highway crack inspecting and sealing robot is another example of the mobile manipulator. It has a gantry-typed Cartesian manipulator with end-effectors for inspection and crack sealing, and a wheeled mobile platform which transports the manipulator. An unmanned excavator is also a mobile manipulator which is being largely used in construction fields. It joins a hydraulic serial manipulator with a caterpillar or tracked mobile base.

This research deals with a wheeled mobile manipulator (WMM) which combines a serial manipulator with a wheeled mobile platform. Generally, a serial manipulator which is operated in a 3-dimmensional space has 6 degrees of freedom (DOF). A wheeled mobile platform has 3 DOF since it can generate a translational and rotational motion in a plane. Therefore a general mobile manipulator which combines two devices listed above comes to have total 9 DOF. However in a 3-dimentional space, only 6 DOF are required to uniquely and perfectly define a motion or a pose of an object. The general mobile manipulator which has 9 DOF contains 3 surplus DOF in aspect of generating 3-dimensional motion. It is called so-tospeak a manipulator with redundancy or a redundant manipulator $^{1-3}$.

A system with redundancy such as the WMM has several advantages. First of all, it has a large flexibility to generate a desired motion of an end-effector since it has a variety of candidates of solution for a motion or a pose. It is advantageous to avoid obsta- 
cles with the surplus DOF. It is possible to evade a singularity which makes the manipulation unstable. Generating a desired motion by operating a 9 DOF system in 3-dimentional space, where only 6 DOF are need to define a motion, there exist not a unique solution but an infinite number of solutions due to the surplus $\mathrm{DOF}^{4,5}$. To choose or find one solution which is optimal to performance requirements among infinite solutions which exist in a redundant system problem is called redundancy resolution ${ }^{6,7}$.

In this paper, a state-space modeling of a WMM is performed, which is a preliminary work to redundancy resolution of the WMM. Due to complexity problem, instead of the general 9 DOF WMM, a reduced manipulator which consists of a differentially driven wheeled mobile platform with two wheels and a reduced serial manipulator with two links and two revolute joints. It is quite straightforward and tedious to expend the reduced WMM to the general system. Firstly, in this paper, a kinematic modeling of the WMM is conducted, which considers only the motion of the system. Then, a dynamic model of the WMM is derived, which deals with both the motion and force of the system. Finally, concluding remarks and further research topics are mentioned.

\section{KINEMATIC MODELING OF WMM}

Fig. 1 shows a schematic diagram of a WMM which is the target system of this research. A body fixed frame $\{x y\}$ attached on the mobile platform is a local coordinate system whose origin is located on the center of mobile platform mass and represented by $R$ in terms of a global coordinate system $\{X Y\}$. The mobile platform is transported by two independently driven wheels attached on both sides. The distance between the center of mobile platform mass and each wheel along the wheel axis direction is defined as $a$. The perpendicular distance from the center of mobile platform mass to the wheel axis is $b$. Considering only the mobile platform, the system can be expressed by the following generalized coordinates with five variables.

$$
\mathbf{q}_{r}=\left[\begin{array}{lllll}
x_{r} & y_{r} & \theta & \phi_{R} & \phi_{L}
\end{array}\right]^{T}
$$

where $\left(x_{r}, y_{r}\right)$ is an absolute position of the center of mass, $\theta$ is an absolute rotation angle of the mobile platform, and $\phi_{R}$ and $\phi_{L}$ are angular displacements of the right and left wheels. Assuming that the mobile platform is a nonholonomic system, there exist three constraints caused by non-slip condition of the wheels. The first constraint is that the lateral direction velocity of the wheels is confined to be zero (Eq. (2)). The second and third constraints are originated from an assumption that no slip happens during forward or backward rolling motion of the wheels
(Eqs. (3) and (4)). These constraints can be formulated as three equations explaining a wheel's behavior and a velocity relationship between the mobile platform and wheels like follows.

$$
-\dot{x}_{r} \sin \theta+\dot{y}_{r} \cos \theta-\dot{\theta} b=0
$$

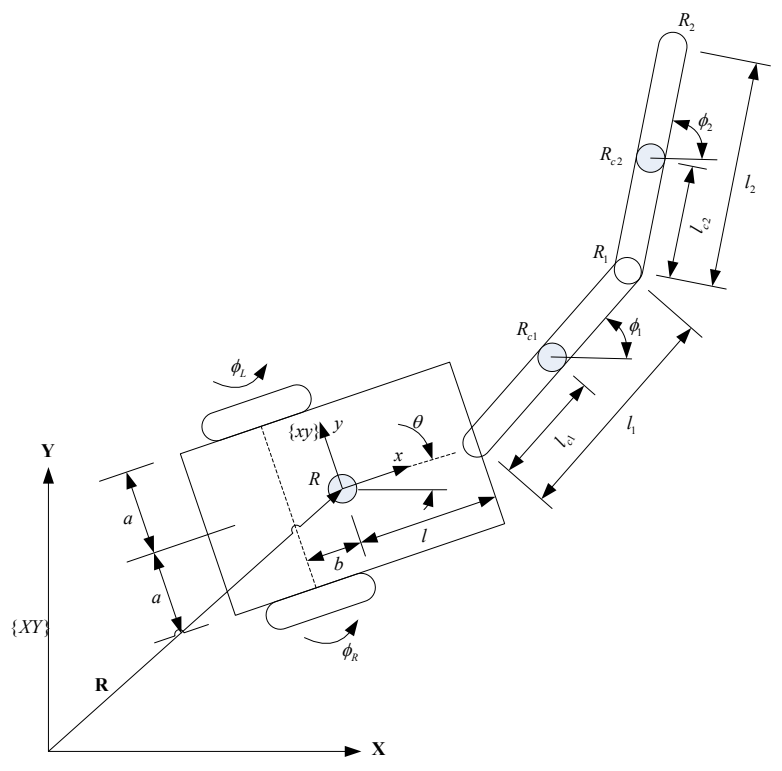

Fig.1. Schematic diagram of the WMM kinematics

$$
\begin{aligned}
& \dot{x}_{r} \cos \theta+\dot{y}_{r} \sin \theta+a \dot{\theta}=r \dot{\phi}_{R} \\
& \dot{x}_{r} \cos \theta+\dot{y}_{r} \sin \theta-a \dot{\theta}=r \dot{\phi}_{L}
\end{aligned}
$$

where $r$ is a radius of the wheel.

As shown in Fig.1, the WMM is configured by a combination of a serial manipulator with two links and two revolute joints and a mobile platform. It is assumed that the attached manipulator generates only 2-dimensional planar motion. In Fig. $1, l_{1}$ and $l_{2}$ are lengths of link 1 and link $2, l_{c 1}$ and $l_{c 2}$ are positions of center of mass of link 1 and link 2 , and $\phi_{1}$ and $\phi_{2}$ are absolute link angles with respect to the global coordinate system $\{X Y\}$. The full set of extended generalized coordinates which add the serial manipulator configuration variables to the mobile platform configuration variables is represented by following vector.

$$
\mathbf{q}=\left[\begin{array}{lllllll}
x_{r} & y_{r} & \theta & \phi_{R} & \phi_{L} & \phi_{1} & \phi_{2}
\end{array}\right]^{T}
$$

Not only the position and velocity kinematic equations of the WMM but also the dynamic equation is derived with above extended generalized coordinates. Three nonholonomic constraints derived above are rearranged like follows.

$\mathbf{C}(\mathbf{q}) \dot{\mathbf{q}}=0$ 
where

$\mathbf{C}_{u \times v=}=\left[\begin{array}{ccccccc}-\sin \theta & \cos \theta & -b & 0 & 0 & 0 & 0 \\ -\cos \theta & -\sin \theta & -a & r & 0 & 0 & 0 \\ -\cos \theta & -\sin \theta & a & 0 & r & 0 & 0\end{array}\right]$

$u(=3)$ is the number of constraints and $v(=7)$ is the number of extended generalized coordinates. From above relationship, a proper null-space matrix $\mathbf{T}$ which satisfies $\mathbf{C T}=0$ can be obtained. In the meanwhile, a velocity vector of the extended generalized coordinates is expressed as a vector with $w(=u-v)$ independent velocities like follows.

$\dot{\mathbf{q}}_{v \times} \boldsymbol{\alpha}=\mathbf{T}_{w \times 1}^{\cdot}$

where

$\dot{\boldsymbol{\alpha}}_{w \times 1}=\left[\begin{array}{llll}\dot{\phi}_{R} & \dot{\phi}_{L} & \dot{\phi}_{1} & \dot{\phi}_{2}\end{array}\right]^{T}$,

$e=r / 2 a$ and

$\mathbf{T}_{v \times w}=\left[\begin{array}{cccc}e(a \cos \theta-b \sin \theta) & e(a \cos \theta+b \sin \theta) & 0 & 0 \\ e(a \sin \theta+b \cos \theta) & e(a \sin \theta-b \cos \theta) & 0 & 0 \\ e & -e & 0 & 0 \\ 1 & 0 & 0 & 0 \\ 0 & 1 & 0 & 0 \\ 0 & 0 & 1 & 0 \\ 0 & 0 & 0 & 1\end{array}\right]$

With respect to the global coordinate system, assuming a planar coordinate of the end-effector is a task-space position $\mathbf{Z}$, a Jacobian matrix $\mathbf{J}$ which relates the joint velocity $\dot{\mathbf{q}}$ of the WMM to the taskspace velocity $\mathbf{Z}$ is defined by Eq.(9).

$\dot{\mathbf{Z}}_{s \times 1}=\mathbf{J} \dot{\mathbf{q}}_{v \times 1}$

where

$\mathbf{J}_{s \times v}=\left[\begin{array}{ccccccc}1 & 0 & -l \sin \theta & 0 & 0 & -l_{1} \sin \theta_{1} & -l_{2} \sin \theta_{2} \\ 0 & 1 & l \cos \theta & 0 & 0 & l_{1} \cos \theta_{1} & l_{2} \cos \theta_{2}\end{array}\right]$,

$S$ is the number of coordinates of the planar position of the end-effector. Substituting Eq.(7) to Eq.(9), a transformed Jacobian $\mathbf{J}_{s \times w}^{*}$ is obtained, which relates the task-space of the end-effector to the independent joint velocities by Eq.(10)

$$
\dot{\mathbf{Z}}_{s \times 1} \boldsymbol{\alpha} \mathbf{J T}^{*} \boldsymbol{\alpha}={ }^{*}{ }_{w \times 1}
$$

\section{DYNAMIC MODELING OF WMM}

In order to derive a governing equation for dynamic analysis for the WMM, Lagrangian formulation is utilized. The WMM is composed of five distinct rigid bodies, mobile platform, right wheel, left wheel, manipulator link 1 and manipulator link 2. It is assumed that two wheels of the mobile platform and two revolute joints of the serial manipulator have ideal linear damping. These four elements are actuators to generate motion and their torques are produced by ideal

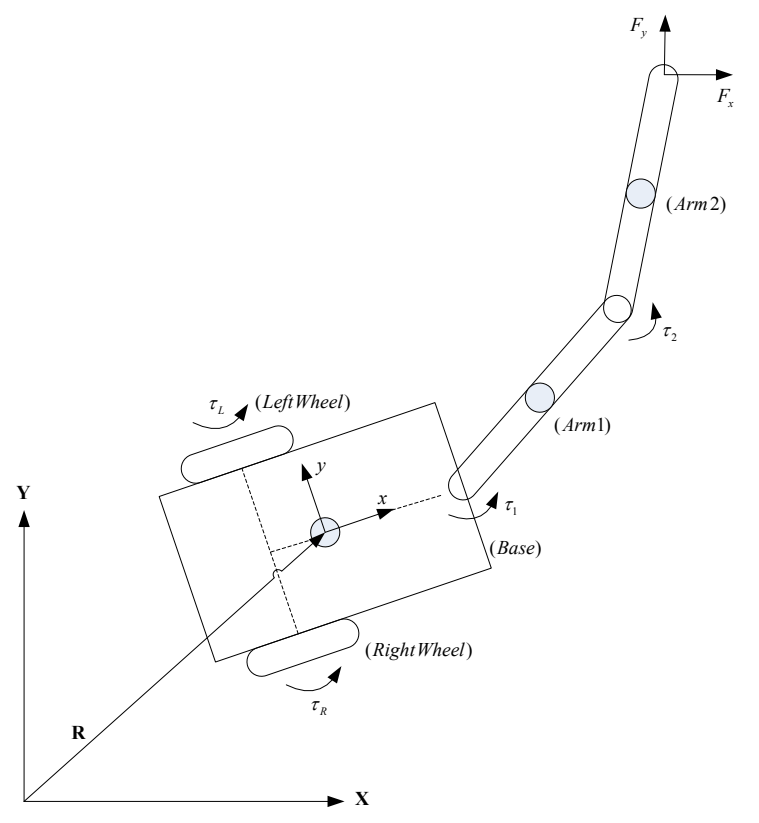

Fig.2. Schematic diagram of the WMM dynamics

Table 1. Dynamic parameters of WMM

\begin{tabular}{ll}
\hline Parameters & \multicolumn{1}{c}{ Description } \\
\hline$I_{w}$ & Wheel inertia \\
$c_{L}, c_{R}$ & Wheel damping \\
$m_{w}$ & Wheel mass \\
$m_{c}$ & Base mass \\
$I_{c}$ & Base inertia \\
$I_{1}$ & Link1 inertia \\
$I_{2}$ & Link2 inertia \\
$m_{1}$ & Link1 mass \\
$m_{2}$ & Link2 mass \\
$d_{1}, d_{2}$ & Joint damping at points $R_{a}$ and $R_{1}$ \\
$\tau_{R}, \tau_{L}$ & Wheel input torque \\
$\tau_{1}, \tau_{2}$ & Motor torque at points $R_{a}$ and $R_{1}$ \\
$F_{x}, F_{y}$ & Environmental force at the end-effector \\
\hline
\end{tabular}

torque-controlled motors. The end-effector attached to the end of the manipulator is connected to the external environment with a pin-joint. Therefore, the interaction between the end-effector and the environment doesn't cause torque but force with $x-y$ components. With the help of aforementioned conditions and Fig.2, the dynamic equation of motion of the WMM is derived like follows. 
$\mathbf{M}(\mathbf{q}) \ddot{\mathbf{q}}+\mathbf{K}(\mathbf{q}, \dot{\mathbf{q}}) \mathbf{r}=\mathbf{F G}_{1} \mathbf{C}+\quad 2 \quad-{ }^{T} \lambda$

$\mathbf{C} \dot{\mathbf{q}}=0$

where $\mathbf{M}$ is a $u \times v$ inertia matrix expressed by the extended generalized coordinates, $\mathbf{K}(\mathbf{q}, \dot{\mathbf{q}})$ represents Coriolis, centrifugal and gravitational forces, $\boldsymbol{\tau}\left(=\left[\begin{array}{llll}\tau_{R} & \tau_{L} & \tau_{1} & \tau_{2}\end{array}\right]^{T}\right)$ means actuator input torques that drive two wheels and two link joints and $\mathbf{F}\left(=\left[\begin{array}{ll}F_{x} & F_{y}\end{array}\right]^{T}\right)$ is an external force which is transferred to the end-effector. $\mathbf{G}_{1}$ matrix maps the active input torques to joint-space and $\mathbf{G}_{2}$ matrix maps the task-space end-effector force $\mathbf{F}$ to joint-space. $\lambda$ is a Lagrangian multiplier. Dynamic parameters are given in Table1 and detailed information of matrix $\mathbf{M}$, $\mathbf{K}, \mathbf{G}_{1}$, and $\mathbf{G}_{2}$ is like follows.

$\mathbf{M}=\left[\begin{array}{ccccccc}h_{1} & 0 & -h_{2} \sin (\theta) & 0 & 0 & -h_{3} \sin \left(\phi_{1}\right) & -h_{4} \sin \left(\phi_{2}\right) \\ 0 & h_{1} & h_{2} \cos (\theta) & 0 & 0 & h_{3} \cos \left(\phi_{1}\right) & h_{4} \cos \left(\phi_{2}\right) \\ -h_{2} \sin (\theta) & h_{2} \cos (\theta) & h_{5} & 0 & 0 & h_{8} & h_{9} \\ 0 & 0 & 0 & h_{7} & 0 & 0 & 0 \\ 0 & 0 & 0 & 0 & h_{7} & 0 & 0 \\ -h_{3} \sin \left(\phi_{1}\right) & h_{3} \cos \left(\phi_{1}\right) & h_{8} & 0 & 0 & h_{6} & h_{11} \\ -h_{4} \sin \left(\phi_{2}\right) & h_{4} \cos \left(\phi_{2}\right) & h_{9} & 0 & 0 & h_{11} & h_{10}\end{array}\right]$

where

$$
\begin{aligned}
& h_{1}=m_{r}+m_{1}+m_{2} \\
& h_{2}=l\left(m_{1}+m_{2}\right) \\
& h_{3}=m_{1} l_{c 1}+m_{2} l_{1} \\
& h_{4}=m_{2} l_{c 2} \\
& h_{5}=l^{2}\left(m_{1}+m_{2}\right)+I_{r} \\
& h_{6}=m_{1} l_{c 1}^{2}+m_{2} l_{1}^{2}+I_{1} \\
& h_{7}=m_{w} r^{2}+I_{w} \\
& h_{8}=l\left(m_{1} l_{c 1}+m_{2} l_{1}\right) \cos \left(-\theta+\phi_{1}\right) \\
& h_{9}=m_{2} l l_{c 2} \cos \left(-\theta+\phi_{2}\right) \\
& h_{10}=m_{2} l_{c 2}^{2}+I_{2} \\
& h_{11}=m_{2} l_{1} l_{c 2} \cos \left(\phi_{1}-\phi_{2}\right) \\
& \mathbf{K}=\left[\begin{array}{llllllll}
K_{1} & K_{2} & K_{3} & c_{R} \dot{\phi}_{R} & c_{L} \dot{\phi}_{L} & K_{6} & K_{7}
\end{array}\right]^{T}
\end{aligned}
$$

where

$$
\begin{gathered}
K_{1}=-l\left(m_{1}+m_{2}\right) \cos (\theta) \dot{\theta}^{2}-\left(m_{1} l_{c 1}+m_{2} l_{1}\right) \phi_{1}^{2} \cos \left(\phi_{1}\right) \\
-m_{2} l_{c 2} \cos \left(\phi_{2}\right) \phi_{2}^{2} \\
K_{2}=-l\left(m_{1}+m_{2}\right) \sin (\theta) \dot{\theta}^{2}-\left(m_{1} l_{c 1}+m_{2} l_{1}\right) \phi_{1}^{2} \sin \left(\phi_{1}\right) \\
-m_{2} l_{c 2} \sin \left(\phi_{2}\right) \phi_{2}^{2} \\
K_{3}=-\left(m_{1} l_{c 1}+m_{2} l_{1}\right) l \dot{\phi}_{1}^{2} \sin \left(-\theta+\phi_{1}\right) \\
-m_{2} l l_{c 2} \dot{\phi}_{2}^{2} \sin \left(-\theta+\phi_{2}\right)-d_{1} \dot{\phi}_{1}+d_{1} \dot{\theta} \\
K_{6}=\left(m_{1} l_{c 1}+m_{2} l_{1}\right) l \dot{\theta}^{2} \sin \left(-\theta+\phi_{1}\right)-d_{1} \dot{\theta}-d_{2} \dot{\phi}_{2} \\
\quad+\left(d_{1}+d_{2}\right) \dot{\phi}_{1}+m_{2} l_{1} l_{c 2} \dot{\phi}_{2}^{2} \sin \left(\phi_{1}-\phi_{2}\right) \\
K_{7}=m_{2} l_{c 2} l \dot{\theta}^{2} \sin \left(-\theta+\phi_{2}\right)-m_{2} l_{c 2} l_{1} \dot{\phi}_{1}^{2} \sin \left(\phi_{1}-\phi_{2}\right) \\
+d_{2} \dot{\phi}_{2}-d_{2} \dot{\phi}_{1}
\end{gathered}
$$

$$
\begin{aligned}
\mathbf{G}_{1} & =\left[\begin{array}{cccc}
0 & 0 & 0 & 0 \\
0 & 0 & 0 & 0 \\
0 & 0 & -1 & 0 \\
1 & 0 & 0 & 0 \\
0 & 1 & 0 & 0 \\
0 & 0 & 1 & -1 \\
0 & 0 & 0 & 1
\end{array}\right] \\
\mathbf{G}_{2} & =\left[\begin{array}{ccc}
0 & 1 & 0 \\
-l \sin (\theta) & l \cos (\theta) \\
0 & 0 \\
& 0 & 0 \\
-l_{1} \sin \left(\phi_{1}\right) & l_{1} \cos \left(\phi_{1}\right) \\
-l_{2} \sin \left(\phi_{2}\right) & l_{2} \cos \left(\phi_{2}\right)
\end{array}\right]
\end{aligned}
$$

\section{CONCLUDING REMARKS}

In this research, kinematic and dynamic modeling of the WMM was performed which is a preliminary work for redundancy resolution. Generalized coordinates were defined on joint-space and a position kinematic equation was derived, which relates the joint variables of the robotic system to task-space variables for an end-effector position. Based on these derivations, a Jacobian that is a velocity relationship of the two spaces was obtained. Finally a dynamic equation of motion was derived, which is a correlation between an external force to the end-effector in terms of taskspace and an internal torque of the robot joints in terms of joint-space. Above governing equations were calculated based on three non-slip constraints that are given under an assumption that the WMM is a nonholonomic system. For further research, detailed optimal selection issues with various redundancy resolution methodologies will be performed using kinematic and dynamic equations obtained by this paper. In addition, to derive some governing equations for omni-directional mobile manipulator (OMM), which is another alternative to replace the nonholomic WMM with, will be studied.

\section{ACKNOWLEDGEMENT}

This work was supported by the Gyeongbuk Science \& Technology Promotion Center(GBSP) grant funded by the Korea government(MEST).

(GBSP -002-111228-002)

\section{References}

1. Hu, Y.M., Guo, B.H., "Modeling and Motion Planning of a Three-link Wheeled Mobile Manipulator", $8^{\text {th }}$ International Conference on Control, Automation, Robotics and Vision, 2004.

2. Boukattaya, M., Jallouli, M., Damak, T. "Dynamic Redundancy Resolution For Mobile Manipulators Using Position Fuzzy Controller", $6^{\text {th }}$ International 
Multi-Conference on Systems, Signals and Devices, 2009.

3. Jallouli, M., Boukattaya, M., Damak, T., "Dynam.lc Redundancy Resolution For Mobile Manipulators With Joints Velocity Limits Avoidance", $5^{\text {th }}$ International Multi-Conference on Systems, Signals and Devices, 2008.

4. Soylu, S., Buckham, B.J., Podhorodeski, R.P., "Redundancy Resolution for Underwater Mobile Manipulators", Ocean Engineering, Vol. 37, pp. 325-343, 2010.

5. Korayem, M.H., Ghariblu, H., "Maximum Allowable Load on Wheeled Mobile Manipulators Imposing Redundancy Constraints", Robotics and Autonomous Systems, Vol. 44, pp. 151-159, 2003.

6. Nenchev, D.N., "Redundancy Resolution through Local Optimization: A Review", Journals of Robotic Systems, Vol. 6(6), pp. 769-798, 1989.

7. Hollerbach, J.M., "Redundancy Resolution of Manipulators through Torque Optimization", IEEE Journal of Robotics and Automation, Vol. RA-3(4), pp. 308-316, 1987. 